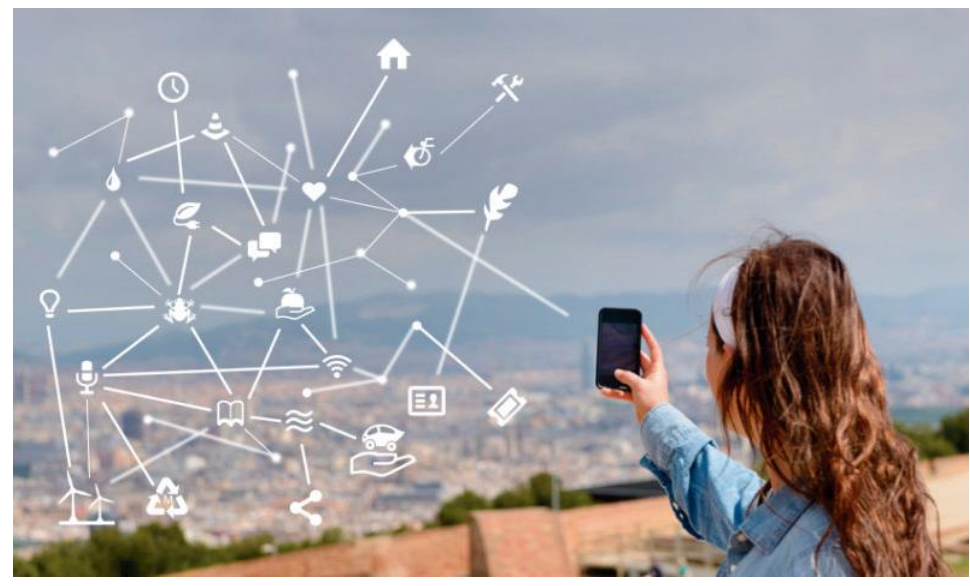

\title{
Mídia territorial Resiliente. Aportes para um debate na Geografia da Comunicação
}

\author{
Resilient territorial media. \\ Contributions to a debate in the Geography of Communication
}

Paulo Celso da Silva ${ }^{1}$

\section{Resumo:}

Os aportes apresentados resultam de reflexões acerca do tema das Smart Cities levado a cabo nos últimos 5 anos, em que as relações mídia e cidade, mídias e tecnologias como estruturadoras do espaço urbano e seus territórios de entorno indicaram a ocorrência de um fenômeno. Isto nos indicou a necessidade de propor e investigar outras possibilidades que se apresentam para o viver contemporâneo e as formas como aplicativos e redes sociais podem ou não auxiliar na superação das dificuldades nos territórios das Smart Cities, a essa dinâmica denominamos de Mídia Territorial Resiliente, entendido aqui como um processo sócio ecológico e não apenas individual. Para tanto, o método de pesquisa é o Mixed Methods e a cidade escolhida, Barcelona.

Palavras-chave: Mídia Territorial Resiliente. Barcelona. Smart Cities. Mixed Methods. Geografia da Comunicação.

\begin{abstract}
:
The contributions presented herein result from reflections about the theme of the Smart Cities, which has been carried out in the last 5 years, in which the relations of media and city, and media and Technologies, as structuring factors to urban space and its surrounding territories, have indicated the occurrence of a phenomenon. This has indicated us the need to propose and investigate other possibilities that appear to contemporary living and the forms; how applicatives and social networks may or may not help to overcome the difficulties in the territories of the Smart Cities; one calls such dynamics Resilient Territorial Media, which is understood here, as a socio-ecological process, instead of an individual one. Thereto, the method of research is Mixed Methods, and the chosen city is Barcelona.
\end{abstract}

Keywords: Resilient Territorial Media. Barcelona; Smart Cities. Mixed Methods. Geography of Communication.

\footnotetext{
${ }^{1}$ Professor Titular do PPG Comunicação e Cultura da Universidade de Sorocaba. Rua Professor Jorge Moisés Betti, 223 - Sorocaba/SP CEP 18095-580. E-mail: paulo.silva@prof.uniso.br.
} 


\section{Mídia Territorial Resiliente}

Inicialmente temos que destacar que entendemos por Mídia Territorial Resiliente os processos urbanos contemporâneos que são baseados ou apoiados em tecnologias de comunicação, podendo ser fixos móveis.

Assim, smartphones, tablets, drones (futuros droids!) redes inalâmbricas, sensores, distribuídos pelos territórios apresentam-se como Mídias Territoriais. Porém, os aplicativos (apps) utilizados em smartphones, as redes sociais (Facebook, Twitter, Instagran, Linkedin, WhatsApp e três centenas mais existentes) e seus usuários, também participam desse processo, aqui considerado sócio ecológico e não apenas individual. Isso porque, a dimensão sócio-ecológica reconhece, de antemão, que "o uso que as pessoas fazem da natureza está embutido no sistema socioeconômico, [...] o conceito de "sistemas socioecológicos" está sendo usado para integrar os processos e componentes socioeconômicos e biofísicos (Machlis et al., 1997; Pickett et al., 1997, Berkes apud BUSCHBACHER, 2014, pág. 11-2)", ao qual acrescentamos também os componentes biomaquínicos, os quais vão muito além da mecânica determinada por seus códigos de execução rígidos e os quais respondem, exatamente, da mesma maneira em todas as situações.

O biomaquínico, ao contrário, age e interage com outras máquinas, seres e situações naturais: conectar com Wi-fi, tirar/enviar fotos, prever o clima, pesar a pressão arterial, afinar um instrumento, utilizando os apps no smartphone, são exemplo simples dessas possibilidades abertas. Como afirmou Guattari, "elas engendram-se umas às outras, selecionam-se, eliminam-se, faz3ndo parecer novas linhas de potencialidades... nunca funcionam isoladamente, mas por agregação ou por agenciamento (GUATTARI \& ROLNIK, 2008, pág. 385)”.

Nesse conceito, também temos de pensar a questão dos territórios, tendo em vista que são sempre plurais quando ampliados para a escala da globalização. Essa escolha, em detrimento de outras, implica em reconhecer, como Milton Santos, como sinônimo de espaço, o espaço geográfico não como sinônimo de território, mas como território usado... Uma perspectiva do território usado conduz a ideia de espaço banal, o espaço de todos, todo o espaço" (SANTOS, 2000, pág. 2).

No bojo da reflexão teórica acerca dos conceitos que demarcam uma geografia da comunicação e as possibilidades de pensar a Mídia Territorial Resiliente, as tecnologias induziram teóricos de várias áreas a afirmar a existência dos não-lugares, 
aqueles que, grosso modo são pontos de passagem, de não-identificação. Entretanto, preferimos continuar com as colocações de Santos (2007, pág. 157) para quem:

A palavra não-lugar, por exemplo, posso continuar utilizando, mas saberei que isso não existe, não há um não-lugar. Um não-lugar é uma metáfora boba e, quando eu a uso, estou suprimindo a capacidade de analisar o que se passa hoje. Porque a globalização é um período no qual os lugares tem um valor que nunca tiveram antes. Nada se faz sem ser em função de um lugar. E as coisas valem hoje, mais do que nunca, em função dos lugares. É por isso que os atores hegemônicos escolhem lugares: as empresas escolhem os seus e deixam os outros para os atores não-hegemônicos. Então a palavra não-lugar corresponde a uma moda, mas não a um modo, ela perturba o processo de análise da realidade.

Assim, o local também dialoga com os territórios nacionais e/ou internacionais muitas vezes conflitando seus interesses econômicos, culturais, sociais com aqueles que as empresas internacionais, normalmente, apoiadas pelos Estados querem impor nos territórios. Isso porque, os agentes com interesses econômicos e financeiros (Estados e empresas internacionais) veem os territórios de maneira homogênea, com suas fronteiras, língua, povo, nacionalidade, direitos; desconsiderando a heterogeneidade do vivido na qual esses atributos políticos e sociais não acontecem de modo standard graças às práticas territoriais imaginárias, concretas, sonhadas pelos diversos agente.

As práticas territoriais vão sugerir também teorizações em torno da desterritorialização e reterritorialização. Guattari, por exemplo, vai lançar mão desses conceitos para analisar a sociedade capitalista, afirmando que o capitalismo "é um bom exemplo de sistema permanente de reterritorialização: as classes capitalistas estão sempre tentando "recapturar" os processos de desterritorialização... das classes sociais" (GUATTARI \& ROLNIK, 2008, pág. 388). Appadurai também analisa essas questões com relação aos Estados-nação e a soberania e os movimentos imigratórios e afirma

$\mathrm{Na}$ Europa contemporânea, de fato, o divórcio entre etnonacionalismo e território toma forma de um reverso perturbador que cada vez mais conforma os movimentos neofascistas da Alemanha, Hungria e outras regiões; seu argumento é simplista: onde quer que os alemães estejam, estamos na Alemanha. Aqui - longe do argumento romântico de que sangue, terra, língua e talvez raça sejam as fundações isomórficas do sentimento de nacionalidade — há o argumento especificamente invertido de que a afiliação étnica gera o território. Assim, o sentimento germânico cria a terra alemã, ao invés de ser seu produto. Esta inversão é uma patologia possível, mas não necessária, da diáspora porque envolve um processo de reterritorialização que antecede o processo de desterritorialização. Trata-se mais exatamente 
da patologia do nacionalismo territorial provocada pela especificidade histórica da ideologia nacional-socialista alemã, pela história particular da formação do Estado na Europa após o império Habsburgo e pela tentadora contiguidade dos alemães "étnicos" separados por fronteiras estatais relativamente recentes. (APPADURAI, 1997, pág. 43)

Entretanto, parece-nos mais acertado considerar a existência da multiterritorialidade, dado que:

Multiterritorialidade aparece como uma resposta a esse processo identificado por muitos como "desterritorialização": mais do que a perda ou o desaparecimento dos territórios, propomos discutir a complexidade dos processos de (re)territorialização em que estamos envolvidos, construindo territórios muito mais múltiplos ou, de forma mais adequada, tornando muito mais complexa nossa multiterritorialidade. Assim, a desterritorialização seria uma espécie de "mito", incapaz de reconhecer o caráter imanente da (multi)territorialização na vida dos indivíduos e dos grupos sociais (Haesbaert, 1994, 2001b, 2004).

Pensar a multiterritorialidade para a Mídia Territorial Resiliente é considerar, como também sugere Haesbaert, a presença de territórios-rede “... na medida em que podem conjugar territórios-zona (manifestados numa escala espacialmente mais restrita) através de redes de conexão (numa escala mais ampla)" (Haesbaert, 2004, pág. 6788), uma vez que os apps são projetados para envio e troca de informações entre pessoas de uma mesma rede, sem, contudo excluir a possibilidade de intercâmbio entre redes. No caso dos aplicativos institucionais, desenvolvidos pelo poder público municipal, para "uso urbano", os cidadãos que possuem smartphones e desejam participar postando informações que alimentam a rede com dados atualizados aos demais conectados, participam de uma rede social sem a necessidade de conhecer os demais usuários. A ligação entre eles dá-se em uma triangulação em movimento que coloca em um mesmo processo APP Usuário/apps/cidade envolvidos pelas redes inalâmbricas. Um esquema possível poderia ser:

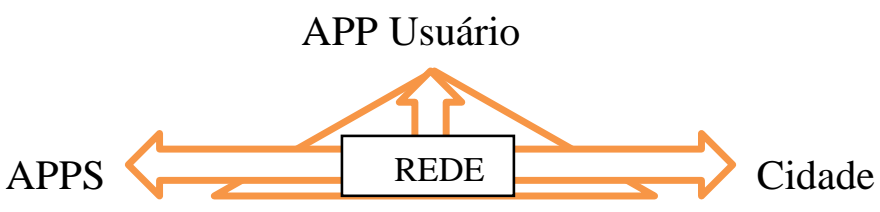

Importante frisar que os Apps são aplicações consideradas simples e podem ser baixadas de um servidor, Google Play, App Store da Apple, por exemplo, grátis ou com 
um preço baixo, eles estão disponíveis para smartphones, com sistemas operacionais Android ou IOS, e alguns para as smartTvs. No Google Play encontramos, para download gratuito, os seguinte aplicativos institucionais de L'Ayuntament de Barcelona (Prefeitura de Barcelona), aqui selecionados pelo critério da valoração dos APPS Usuários alguns deles:

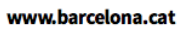

Apps municipals

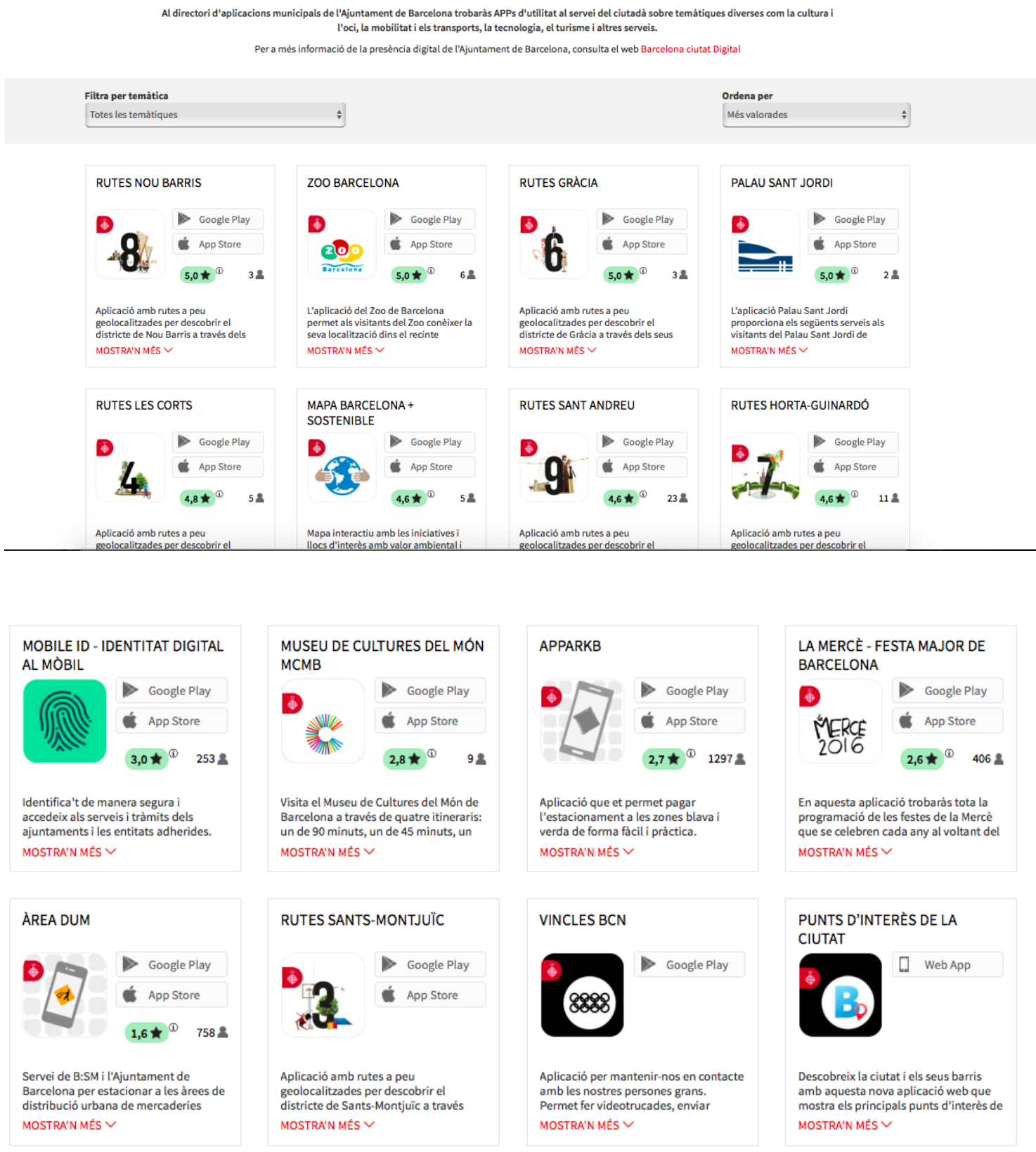

Figure 1 APPS do Ayuntament de Barcelona. Fonte https://ajuntament.barcelona.cat/apps/ca/ 
Dos aplicativos, destacamos a Bústia Ciutadana/Buzón Ciudadano (versão Catalã e Versão Castelhana), um aplicativo para o cidadão relatar o que está acontecendo em sua cidade, solicitar providências para algum problema.

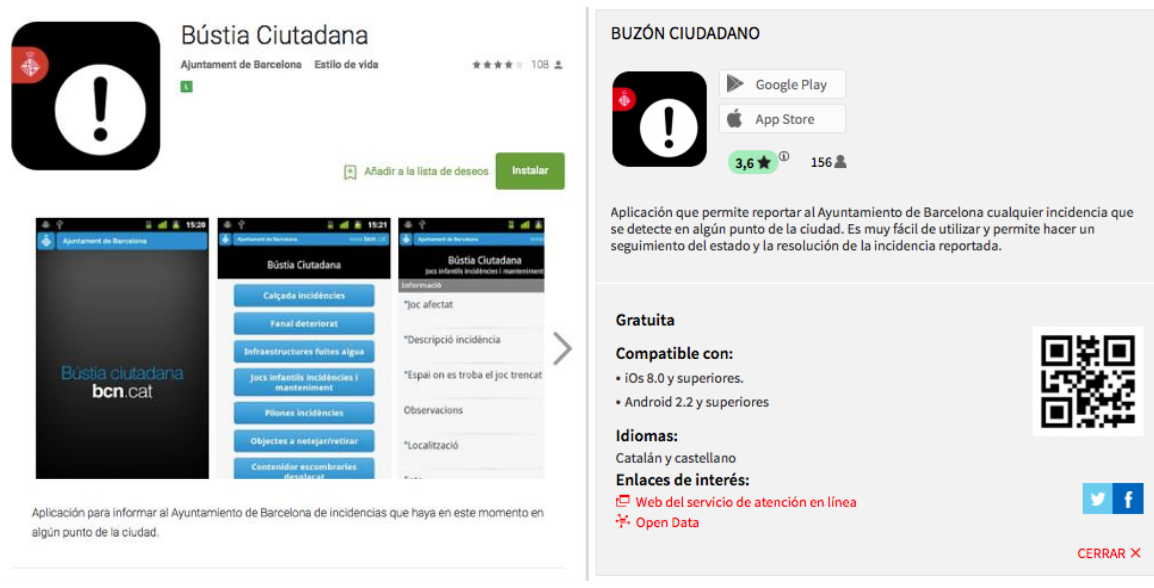

OPINIONES

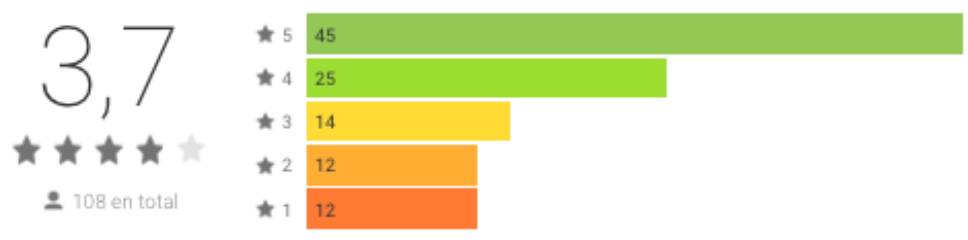

Conforme a interação das pessoas, o aplicativo, que teve o primeiro comentário em 2011, “Que bom!!! Funciona muito bem no Nexus S, recomendo!”. lembrando que Nexus S foi um smartphone lançado em 2010 da parceria da Samsung com o Google e fabricado até 2016, considerado como 'puro Android', por não sofrer modificações do fabricante.

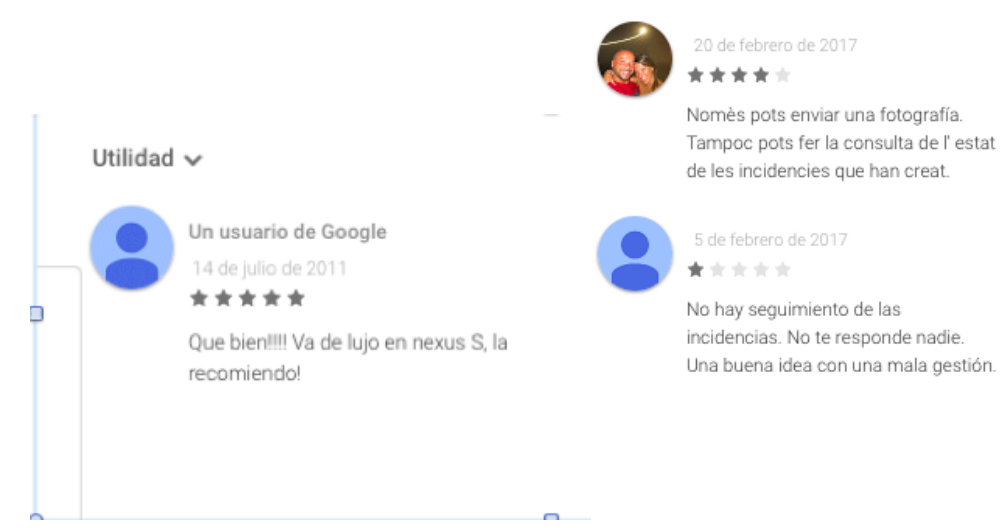

Figure 2 Primeiro comentário do Apps, 14/07/2011. E comentários do ano de 2017. Fonte: https://play.google.com/store/apps/details?id=es.tempos21.bustia\&hl=es 
Os últimos comentários encontrados em 5 e 20 de fevereiro 2017, respectivamente, reclamavam das funcionalidades do Apps. O primeiro valorando com apenas uma estrela apontava que, "Não há seguimento das incidências. Ninguém responde a você. Uma boa ideia com uma má gestão”. E o segundo , mesmo avaliando com 4 estrelas reclamava, "Não se pode enviar uma fotografia. Tampouco podemos fazer uma consulta da situação das incidências que enviamos",

Nas estatísticas disponíveis desse aplicativo, vemos que ele foi atualizado, na versão 2.6.1, em 05 de abril de 2017 com o intuito de "corrigir erros", entre eles "ler o conteúdo de fotos, multimídia e arquivos armazenados em USB, realizar fotos e vídeos" e as descargas estavam entre 5.000 e 10.000.

Solicitamos na "Oficina d'Atenció Ciutadana", responsável pelo aplicativo, dados mais detalhados do uso, mas ainda não obtivemos a resposta. Contudo, a leitura dos comentários parece indicar que existe uma preocupação, da Prefeitura, em responder as questões indicadas pelos cidadãos. é o que também indica o "MESURA DE GOVERN", o Pla Barcelona Ciutat Digital, lançado em outubro de 2016, com o intuito de "ir além da mera construção de SmartCity" e sugere um plano de ação:

Estas Medidas incluem as diretrizes governamentais que sustentam o Plano Municipal de Barcelona Digital, um plano vivo que será moldado e detalhado em seu curso. Este Plano transversal tem como objetivo ser um guarda-chuva que pretende fazer uma política de tecnologia coerente e consistente na cidade, com uma visão que inclui iniciativas e estratégias implementadas em diferentes áreas. Ela é organizada sob os seguintes prismas: Governo, Cidade, Tecido sócio econômico e digital e ecossistema de inovação local, Cidadania (MESURA DE GOVERN... págs. 3-4).

Em termos de políticas públicas para a Cidade digital, percebe-se uma transformação nos parâmetros de comunicação urbana. Em 2001, quando dos primeiros passos do projeto de inovação tecnológica 22@BCN, a proposta conceitual da cidade era ser multifuncional, heterogênea e diversa, compacta e densa (SILVA, pág.55). A proposta para 2017-2020 amplia os conceitos para “...uma cidade aberta, equitativa, democrática e circular, que aproveita as oportunidades da inovação com base em dados para melhorar a cidade e a vida dos cidadãos” (MESURA DE GOVERN..., pág. 3). O conceito de circular refere-se à proposta de um modelo urbano sustentável e eficiente, encarando “..os problemas mais urgentes no ambiente urbano: gestão de resíduos, energia, mobilidade, habitação, a luta contra a exclusão social, o emprego e o 
envelhecimento da população, para garantir uma melhor qualidade de vida e mais equitativa" (BARCELONA CIUTAT DIGITAL..). Tudo isso com base no aperfeiçoamento das tecnologias de uso cidadão.

Estudo feito, em 2016, pela Fundació Mobile (Mobile World Capital), contratada pela prefeitura de Barcelona, vai indicar as Brechas Digitais na cidade. Contudo, antes dos dados, cabe uma rápida explanação no método de análise aqui utilizado.

\section{Mixed Methods}

A metodologia utilizada está baseada no Método Misto (Mixed Methods) proposto por John Creswell (2007). Entendemos que para trabalhar com dados qualitativos e quantitativos configura-se como uma opção satisfatória para a comunicação, também pelas possibilidades que o enriquecimento qualitativo e quantitativo nos oferecem.

Creswell desenvolve diversas Estratégias alternativas e modelos gráficos, a saber: Estratégia explanatória sequencial, Estratégia exploratória sequencial, Estratégia transformadora sequencial, Estratégia de triangulação concomitante, Estratégia aninhada concomitante, Estratégia transformadora concomitante (2007, p. 211-225), e aqui vamos desenvolver a que nos parece mais satisfatória a Estratégia Transformadora Concomitante.

Tal Estratégia é norteada nos estudos em que objetivos se sobrepõe ao uso dos métodos e ocorre com a coleta dos dados (2007, p. 32)

...a técnica transformadora concomitante é guiada pelo uso que o pesquisador faz de uma perspectiva teórica específica. Essa perspectiva pode ser baseada em ideologias como teoria crítica, reivindicatória, pesquisa participatória ou em uma estrutura conceitual ou teórica. Essa perspectiva é refletida no objetivo ou nas questões de pesquisa do estudo. É a força condutora por trás de todas as escolhas metodológicas, como definição do problema, identificação do projeto e das fontes de dados, análise, interpretação e comunicação de resultados durante todo o processo de pesquisa. A escolha de um modelo concomitante (seja de triangulação, seja de projeto aninhado) é feita para facilitar essa perspectiva. Por exemplo, o projeto pode ser aninhado para que diversos participantes tenham voz no processo de mudança de uma organização que esteja sendo estudada principalmente de forma quantitativa. Isso pode envolver a triangulação de dados quantitativos e qualitativos para melhor convergir as informações para gerar evidência para uma desigualdade de políticas em uma organização. Assim, o modelo transformador concomitante pode assumir as características de projeto de uma 
triangulação ou de um método aninhado. Ou seja, os dois tipos de dados são coletados ao mesmo tempo durante uma fase de coleta de dados e podem ter prioridade igual ou desigual. A integração desses dados diferentes ocorre mais frequentemente durante a fase de análise, embora a integração durante a fase de interpretação seja urna variação possível. Corno o modelo transformador concomitante compartilha características com as técnicas de triangulação e aninhada, ele também compartilha seus pontos fortes e pontos fracos específicos. No entanto, esse modelo tem a vantagem adicional de posicionar a pesquisa de métodos mistos dentro de urna estrutura transformadora, o que pode torná-la especialmente atraente para aqueles pesquisadores qualitativos ou quantitativos que já usam urna estrutura transformadora para guiar sua investigação.

Assim os dados quantitativos, aqueles que são mensuráveis, dedutíveis e objetivos, estariam relacionados à População, uso dos Apps, mobilidade, pobreza, participação cidadã, serviços. Os dados Qualitativos, ligados aos fenômenos sociais como algo construído estariam relacionados aos levantamentos das políticas públicas voltadas ao meio ambiente e à cidadania, ao papel da organização de bairros nas decisões políticas e à qualidade dos Apps de uso público (estas medidas e indicadas pelos usuários). Com isso os estudos atenderiam às três ordens da investigação: a $1^{\text {a }}$. Ordem, da descrição; $2^{\mathrm{a}}$. Ordem, da reflexão e $3^{\mathrm{a}}$. Ordem, dos processos.

\section{Barcelona Digital em dados}

Dado a quantidade de dados no estudo das Brechas digitais de Barcelona, aqui utilizaremos os levantamentos quantitativos referentes aos bairros e, quando necessário, cruzaremos com outras informações que sejam pertinentes para indicar a Mídia Territorial Resiliente.

Assim, o primeiro dado significativo foi no tocante ao uso da internet, considerado para os 40 grandes bairros: 


\begin{tabular}{|c|c|c|c|}
\hline \multicolumn{4}{|c|}{ TAULA 3. DETALLE DE GRANDES BARRIOS Y BARRIOS DE LA CIUDAD DE BARCELONA } \\
\hline \multicolumn{2}{|r|}{ GRAN BARRIO } & \multicolumn{2}{|r|}{ BARRIO } \\
\hline 1 & el Raval & 1 & el Raval \\
\hline 2 & el Barri Gòtic & 2 & el Barri Gòtic \\
\hline 3 & la Barceloneta & 3 & la Barceloneta \\
\hline 4 & Sant Pere, Santa Caterina i la Ribera & 4 & Sant Pere, Santa Caterina i la Ribera \\
\hline 5 & el Fort Pienc & 5 & el Fort Pienc \\
\hline 6 & la Sagrada Família & 6 & la Sagrada Família \\
\hline 7 & la Dreta de l'Eixample & 7 & la Dreta de l'Eixample \\
\hline 8 & I'Antiga Esquerra de l'Eixample & 8 & I'Antiga Esquerra de l'Eixample \\
\hline 9 & la Nova Esquerra de l'Eixample & 9 & la Nova Esquerra de l'Eixample \\
\hline 10 & Sant Antoni & 10 & Sant Antoni \\
\hline 11 & el Poble Sec & 11 & el Poble Sec \\
\hline \multirow{2}{*}{12} & \multirow{2}{*}{ la Marina } & 12 & la Marina del Prat Vermell \\
\hline & & 13 & la Marina de Port \\
\hline \multirow{3}{*}{13} & \multirow{3}{*}{$\begin{array}{l}\text { la Font de la Guatlla, Hostafrancs i la } \\
\text { Bordeta }\end{array}$} & 14 & la Font de la Guatlla \\
\hline & & 15 & Hostafrancs \\
\hline & & $17^{16}$ & $\begin{array}{c}\text { la Bordeta } \\
\text { Sants - Badal }\end{array}$ \\
\hline 14 & Sants i Sants-Badal & 18 & Sants \\
\hline 15 & les Corts & 19 & les Corts \\
\hline 16 & la Maternitat i Sant Ramon & 20 & la Maternitat i Sant Ramon \\
\hline 17 & Pedralbes & 21 & Pedralbes \\
\hline \multirow{3}{*}{19} & \multirow{3}{*}{ Sarrià, les Tres Torres i Vallvidrera } & 22 & Vallvidrera, el Tibidabo i les Planes \\
\hline & & 23 & Sarrià \\
\hline & & 24 & les Tres Torres \\
\hline \multirow{2}{*}{20} & \multirow{2}{*}{ Sant Gervasi-la Bonanova i el Putxet } & 25 & Sant Gervasi - la Bonanova \\
\hline & & 27 & el Putxet i el Farró \\
\hline 21 & Sant Gervasi-Galvany & 26 & Sant Gervasi - Galvany \\
\hline \multirow{3}{*}{22} & \multirow{3}{*}{ Vallcarca, el Coll i la Salut } & 28 & Vallcarca i els Penitents \\
\hline & & 29 & el Coll \\
\hline & & 30 & la Salut \\
\hline 23 & la Vila de Gràcia & 31 & la Vila de Gràcia \\
\hline 24 & el Camp d'en Grassot i Gràcia Nova & 32 & el Camp d'en Grassot i Gràcia Nova \\
\hline \multirow{2}{*}{25} & \multirow{2}{*}{ el Guinardó } & 33 & el Baix Guinardó \\
\hline & & 35 & el Guinardó \\
\hline 26 & el Carmel i Can Baró & 34 & Can Baró \\
\hline \multirow{2}{*}{26} & \multirow{2}{*}{ el Carmel i Can Baró } & $\overline{34}$ & Can Baró \\
\hline & & 37 & el Carmel \\
\hline & & 38 & la Teixonera \\
\hline & & 39 & Sant Genis dels Agudells \\
\hline 27 & Los Barrios de la Vall d'Hebron & 40 & Montbau \\
\hline & & 41 & la Vall d'Hebron \\
\hline & & 42 & la Clota \\
\hline 28 & Hortaj la Font d'en Faraues & 36 & la Font d'en Fargues \\
\hline & & 43 & Horta \\
\hline 26 & el Carmel i Can Baró & 37 & el Carmel \\
\hline & & 38 & la Teixonera \\
\hline & & 39 & Sant Genis dels Agudells \\
\hline 27 & Los Barrios de la Vall d'Hebron & 40 & Montbau \\
\hline & & 41 & la Vall d'Hebron \\
\hline & & 42 & la Clota \\
\hline 28 & Horta i la Font d'en Fargues & 36 & la Font d'en Fargues \\
\hline & & 43 & Horta \\
\hline
\end{tabular}




\begin{tabular}{|c|c|c|c|}
\hline & GRAN BARRIO & & BARRIO \\
\hline \multirow{4}{*}{29} & \multirow{4}{*}{$\begin{array}{l}\text { Vilapicina, Porta, el Turó de la Peira i Can } \\
\text { Peguera }\end{array}$} & 44 & Vilapicina i la Torre Llobeta \\
\hline & & 45 & Porta \\
\hline & & 46 & el Turó de la Peira \\
\hline & & 47 & Can Peguera \\
\hline \multirow{3}{*}{30} & \multirow{3}{*}{ la Guineueta, Verdun i la Prosperitat } & 48 & la Guineueta \\
\hline & & 51 & Verdun \\
\hline & & 52 & la Prosperitat \\
\hline \multirow{3}{*}{31} & \multirow{3}{*}{ Canyelles, les Roquetes i la Trinitat Nova } & 49 & Canyelles \\
\hline & & 50 & les Roquetes \\
\hline & & 53 & la Trinitat Nova \\
\hline \multirow{3}{*}{32} & \multirow{3}{*}{ Torre Baró, Ciutat Meridiana i Vallbona } & 54 & Torre Baró \\
\hline & & 55 & Ciutat Meridiana \\
\hline & & 56 & Vallbona \\
\hline \multirow{3}{*}{33} & \multirow{3}{*}{$\begin{array}{l}\text { la Trinitat Vella, Baró de Viver i el Bon } \\
\text { Pastor }\end{array}$} & 57 & la Trinitat Vella \\
\hline & & 58 & Baró de Viver \\
\hline & & 59 & el Bon Pastor \\
\hline 34 & Sant Andreu & 60 & Sant Andreu \\
\hline \multirow{3}{*}{35} & \multirow{3}{*}{ la Sagrera, el Congrés i Navas } & 61 & la Sagrera \\
\hline & & 62 & el Congrés i els Indians \\
\hline & & 63 & Navas \\
\hline \multirow{2}{*}{36} & \multirow{2}{*}{ el Clot i el Camp de l'Arpa } & 64 & el Camp de l'Arpa del Clot \\
\hline & & 65 & el Clot \\
\hline \multirow{2}{*}{37} & \multirow{2}{*}{ el Parc, la Llacuna i la Vila Olimpica } & 66 & el Parc i la Llacuna del Poblenou \\
\hline & & 67 & la Vila Olímpica del Poblenou \\
\hline \multirow{2}{*}{38} & \multirow{2}{*}{ el Poblenou i Diagonal Mar } & 68 & el Poblenou \\
\hline & & 69 & Diagonal Mar i el Front Marítim del Poblenou \\
\hline \multirow{2}{*}{39} & \multirow{2}{*}{ el Besòs, el Maresme i Provençals } & 70 & el Besòs i el Maresme \\
\hline & & 71 & Provençals del Poblenou \\
\hline \multirow{2}{*}{40} & \multirow{2}{*}{ Sant Martí, la Verneda i la Pau } & 72 & Sant Martí de Provençals \\
\hline & & 73 & la Verneda i la Pau \\
\hline
\end{tabular}

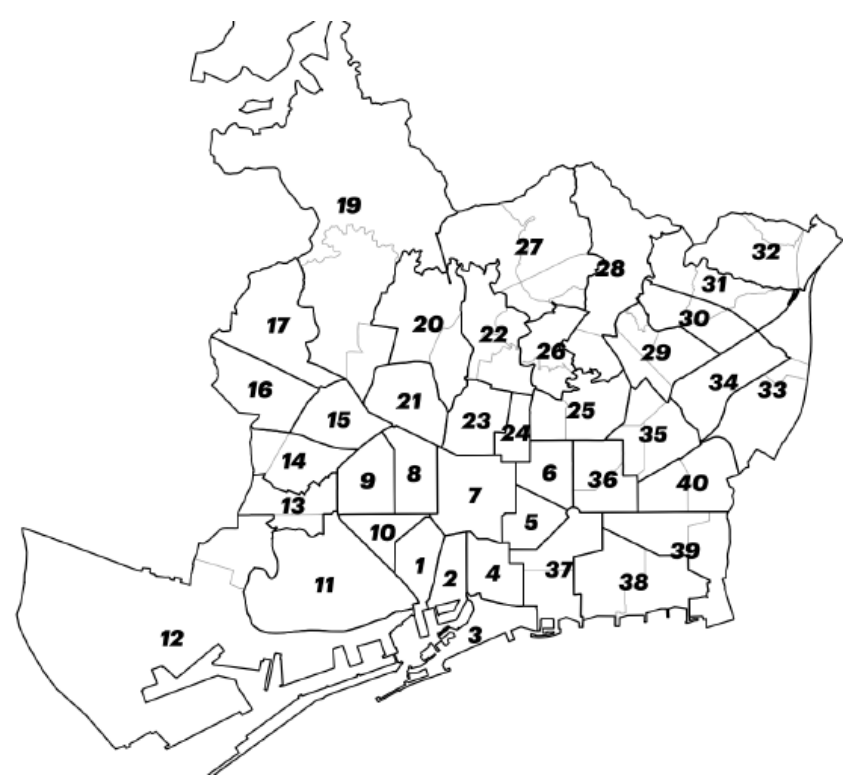

Figure 3 Divisão territorial segundo a renda e os grandes bairros

O Estudo indica que, com base nos resultados de acesso e uso da internet, existe uma brecha territorial na Cidade Condal, com diferenças, no item da conexão em casa, que chegam a 34 pontos: Les Corts, 96\%; Torre Baró, 62\%. Quando a conexão é feita 
por meio de smartphones a diferença chega a 40 pontos: Dreta de l'Eixample, $94 \%$ y de Torre Baró, 55\%. As mesmas diferenças foram verificadas no uso de internet entre os grandes bairros, isso em uma cidade em que 9 em 10 barceloneses se conectam diariamente a rede (LA BRECHA DIGITAL..., pág. 18). O estudo também aponta que a brecha digital é mais acentuada nos grupos com mais de 65 anos de idade e que, considerando gênero apenas masculino e feminino (como aconteceu no estudo), não determina acesso e uso visto que $77 \%$ de homens e mulheres possuem smartphones. Contudo, o uso é diferenciado com os homens acessando mais conteúdos de atividades comerciais e as mulheres as atividades ligadas à saúde. (LA BRECHA DIGITAL..., 2016, pág. 17).

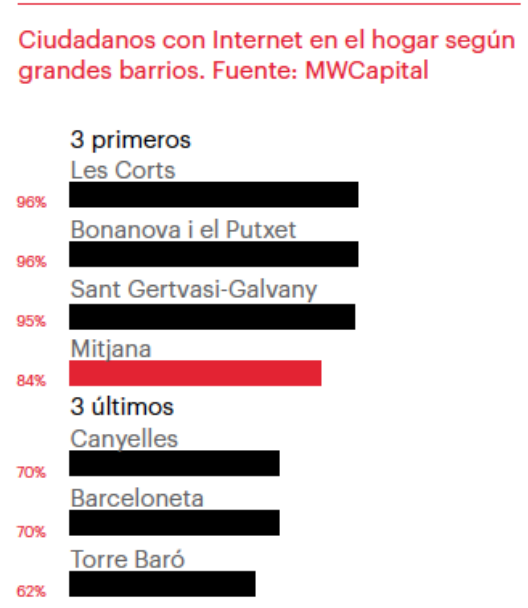

Figure 4 Cidadãos com internet em casa segundo grandes Bairros. Fonte MWCapital

Mas, no item da renda dos bairros, a brecha digital aparece de maneira mais destacada e crítica. Nos bairros de renda mais alta, as pessoas utilizam a internet para encontrar notícias da atualidade, fazer gestões bancarias e compras online, com frequência muito maior que nos bairros de rendas baixas. No que respeita as variáveis, gênero, idade, nível educativo e emprego, nos bairros de baixa renda ocorre uma menor incidência no uso da internet pelas mulheres entre 65 e 74 anos, com baixo nível educativo, donas de casa, domésticas ou desempregadas.

Nos bairros de baixa renda, 53\% das pessoas de 65 a 74 anos não possuem internet em suas casas, já nos bairros de alta renda, 88\% de pessoas com a mesma idade, contam com internet em suas casas.

O nível educativo é considerado pelo estudo como a brecha digital mais importante nos bairros, exceto nos de renda alta, tanto para o acesso quanto para o uso. Os números indicam que em bairros de baixa renda, 94\% das pessoas com alto nível educacional têm conexão à internet em casa; esse número cai para $60 \%$ das pessoas 
com baixo nível de escolaridade; em bairros de alta renda $83 \%$ das pessoas com baixo nível de educação têm Internet em casa, o número chega a 95\% para os de alto nível educacional (LA BRECHA DIGITAL..., 2016, pág. 19). Conclui o estudo:

Finalmente, a ocupação também demonstra ser uma brecha importante em bairros de nível de renda baixa. A exclusão digital é dada, especialmente entre os desempregados, ocupados em tarefas domésticas e aposentados; perfis menos digitais em bairros de baixa renda. Por outro lado, os alunos e ocupados mostram um alto nível de digitalização, independentemente da renda do bairro (LA BRECHA DIGITAL..., 2016, pág. 19).
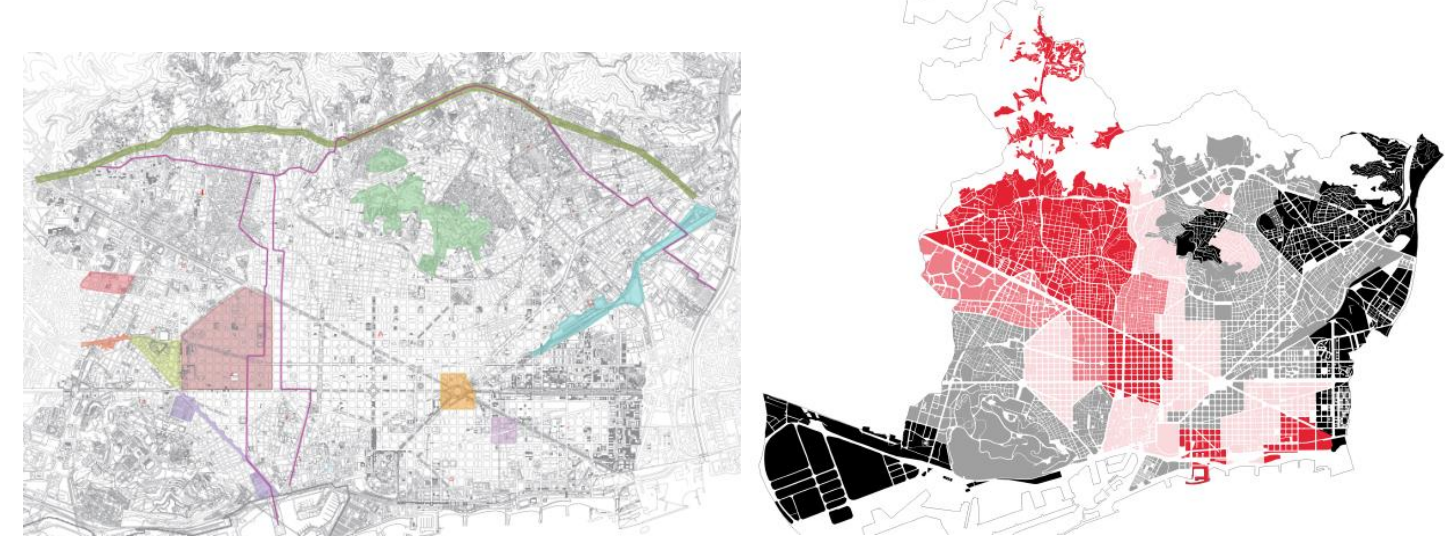

Figure 5 Cruzamento das informações de renda familiar por bairro e participação cidadã em projetos municipais

Para finalizar, os dois mapas acima indicam a Distribuição da Renda Familiar pelos bairros de Barcelona sendo o vermelho mais forte renda superior a 140 RFD5 e a preta renda baixa inferior a 60 RDF1 (sendo cada RDF $=100$ ). Ao lado, o Plano, Projetos e Linhas de Trabalho com participação cidadã - Dezembro de 2015. O que se mostra imediatamente ao pesquisador, é que as frentes de atuação estão nas áreas de renda média e a linha verde, que corresponde ao eixo Ronda de Dalt (liga uma área pobre e uma rica, assim como as linhas magenta que correspondem a quarta fase da nova rede de ônibus, a qual também liga o eixo mar - montanha.

Assim, tanto as ações oficiais quanto a maior participação dos cidadãos, opinando nos planos e projetos estão relacionados com a renda, de média para alta.

\section{Conclusão}

Em virtude da dimensão do artigo, as informações e dados foram reduzidas ao essencial para indicarmos os aportes de uma discussão maior, do tema da Mídia Territorial Resiliente, na Geografia da Comunicação.

Ainda assim, é possível afirmar que as smart cities, e Barcelona em especial, preparam-se para oferecer diferenciais para o mercado investidor, mas também para os 
cidadãos que ali vivem e convivem com as contradições que os modelos de gestão urbano-comunicacionais prometem e aplicam no cotidiano. Nesse sentido, o estudo acerca da Brecha digital na cidade, não aborda um problema ligado ao turismo e as ofertas de quartos na cidade, em casas e apartamentos das áreas centrais (bairro Gòtic, Raval) e beira mar (Barceloneta) postados no site e aplicativo do AIRBNB, mesmo com as regras turísticas existentes.

O ponto positivo, no universo da conexão e uso, é Barcelona estar entre as principais cidades europeias gerando um volume diário de atividade em rede que evidenciam o grau de penetração digital na cidadania (LA BRECHA DIGITAL..., 2016, pág. 28) o que pode favorecer o desenvolvimento da cidadania e ampliação das possibilidades de resiliência dos moradores.

No que respeita à utilização do Mixed Method neste trabalho, entendemos que foi possível mostrar como a triangulação entre os dados qualitativos e quantitativos, coletados conforme a pesquisa se desenvolve e ambos com prioridades variáveis dependendo do momento em que as buscas e reflexões ocorrem.

Por fim, a Mídia Territorial Resiliente, enquanto processo sócio ecológico e não apenas individual, amplia o papel dos meios, assim a sua responsabilidade na transformação necessária da comunicação urbana para um ambiente integrado de tecnologia/natureza não linear, em que as contradições colocam-se como obrigatórias para que o movimento social possa acontecer e avançar rumo a uma maior distribuição dos benefícios que as Smart cities possam oferecer.

Analisar as Smart cities pelo viés da Mídia Territorial Resiliente reforça ainda mais o fato de que não existem modelos prontos, estandardizados para todos os territórios ou cidades. O desafio continua sendo construir uma smart city adequada para aquele território e população.

\section{REFERÊNCIAS}

AJUNTAMENT DE BARCELONA. Aplicativos. Disponível em < https://play.google.com/store/apps/developer?id=Ajuntament+de+Barcelona> Acesso em 02 Jun. 2017.

APPADURAI, A. Soberania sem territorialidade. Notas para uma geografia pósnacional. Revista Novos Estudos CEBRAP, n. 49, 1989, págs. 33-49. Também disponível em <https://edisciplinas.usp.br/pluginfile.php/363238/mod_resource/content /0/8-Appadurai-notas_para_uma_geografia.pdf > Acesso em 10 de Mai 2017.

BARCELONA CIUTAT DIGITAL. Disponível em < http://ajuntament.barcelona.cat /estrategiadigital/es> Acesso em 25 de Maio 2017. 
BUSCHBACHER, R A teoria da resiliência e os sistemas socioecológicos: como se preparar para um futuro imprevisível? IPEA, Boletim Regional, Urbano e Ambiental | 09 | Jan.- Jun. 2014.

GUATTARI, F. \& ROLNIK. Micropolítica. Cartografias do Desejo. Petrópolis: Vozes, 2008.

HAESHAERT, R. O Mito da Desterritorialização. Rio de Janeiro: Bertrand Brasil, 2004.

HAESHAERT, R. Da desterritorialização à multiterritorialidade in Anais do X Encontro de Geógrafos da América Latina - 20 a 26 de março de 2005 - Universidade de São Paulo, págs. 6774 - 6792.

MESURA DE GOVERN: Transició cap a la sobirania tenològica. Pla Barcelona Ciutat Digital, octubre de 2016. Disponível em <http://ajuntament.barcelona.cat/ estrategiadigital/uploads/Pla_Ciutat_Digital_MdGovern.pdf > Acesso em 25 Mai 2017.

LA BRECHA DIGITAL en la ciudad de Barcelona. Disponível em <www.mobileworldcapital.com/escletxa-digital> Acesso em 25 Mai. 2017.

MOBILE WORLD CAPITAL. Disponível em <http://www.mobileworldcapital.com /escletxa-digital/index_cas.php\#8thPage> Acesso em 25 Mai 2017.

SANTOS, M. et. al. O papel da geografia. Um manifesto. XII Encontro Nacional de Geógrafos. Florianópolis, julho, 2000.

SANTOS, M. Espaço, mundo globalizado, pós-modernidade IN Milton Santos, Encontros. A arte da Entrevista. Organização Maria Angela P. Leite Rio de Janeiro: Beco do Azougue, 2007.

SILVA, P. C. \& SILVA, N.M.P. POBLENOU: território @ de Barcelona. Projeto 22@BCN Estudos e considerações. Itu: Ottoni, 2006. 


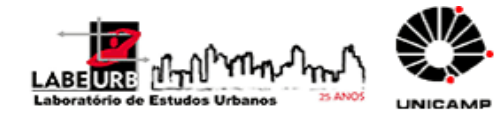

\section{Para citar essa obra:}

SILVA, Paulo Celso da. Mídia territorial Resiliente. Aportes para um debate na Geografia da Comunicação. In: RUA [online]. no. 23. Volume 2, p. 373 - 387 - e-ISSN 2179-9911 Novembro/2017. Consultada no Portal Labeurb - Revista do Laboratório de Estudos Urbanos do Núcleo de Desenvolvimento da Criatividade.

http://www.labeurb.unicamp.br/rua/

Capa: Disponível em: <https://www.citizenlab.co/wp-content/uploads/2016/11/proj-node-124-11024x474.jpg>

Laboratório de Estudos Urbanos - LABEURB

Núcleo de Desenvolvimento da Criatividade - NUDECRI

Universidade Estadual de Campinas - UNICAMP

http://www.labeurb.unicamp.br/

Endereço:

LABEURB - LABORATÓRIO DE ESTUDOS URBANOS

UNICAMP/COCEN / NUDECRI

CAIXA POSTAL 6166

Campinas/SP - Brasil

CEP 13083-892

Fone/ Fax: (19) 3521-7900

Contato: http://www.labeurb.unicamp.br/contato 